\title{
Enseñar las unidades fraseológicas a través de la literatura en el aula de ELE
}

\section{Teaching phraseological units through literature in the ELE classroom}

\section{Rosaria Minervini}

Universidad de Salerno

minervini@unisa.it

ORCID ID: https://orcid.org/0000-0003-0739-0358

DOI: $10.17398 / 1988-8430.34 .83$

Minervini, R. (2021). Enseñar las unidades fraseológicas a través de la literatura en el aula de ELE. Tejuelo, 34, 83-110.

Doi: https://doi.org/10.17398/1988-8430.34.83 
Resumen: Las unidades fraseológicas representan un componente esencial para el desarrollo de las competencias léxica y comunicativa de los aprendientes, y su enseñanza en el aula de español como lengua extranjera ocupa un lugar cada vez más relevante. En este trabajo, se presentan algunas actividades de aula para enseñar estas unidades a través de la literatura y, en concreto, mediante la novela $\mathrm{Sin}$ noticias de Gurb, de Eduardo Mendoza. Después de una parte teórica en la que se intentará aclarar la importancia de la fraseología como componente imprescindible del acervo lingüístico de los aprendientes y el papel que la literatura puede desarrollar en el proceso de enseñanza/aprendizaje de estas unidades, en una segunda parte se propone una serie de actividades concretas cuyo objetivo es ayudar a desarrollar la competencia fraseológica a través de la novela mencionada. Pensamos que la literatura ayuda a contextualizar y, por lo tanto, a entender mejor las unidades fraseológicas, así como contribuye a diseñar la estrecha relación que existe entre fraseología y cultura. Nuestro propósito es, en definitiva, impulsar la enseñanza de la fraseología en el aula de ELE a través de la literatura.

Keywords: fraseología; literatura; ELE; enseñanza/aprendizaje;

Mendoza.
Abstract: Phraseological units represent an essential component for the development of the lexical and communicative competence of learners and their teaching in the classroom of Spanish as a foreign language occupies an increasingly relevant place. This work presents some classroom activities to teach these units through literature and, specifically, through the novel Sin noticias de Gurb by Eduardo Mendoza. After a theoretical part in which we will try to clarify the importance of phraseology as an essential component of the linguistic heritage of learners and the role that literature can develop in the teaching/learning process of these units, in a second part we propose a series of concrete activities whose objective is to help develop phraseological competence through the aforementioned novel. We believe that literature helps to contextualize and, therefore, to better understand phraseological units as well as helping to design the close relationship that exists between phraseology and culture. Our purpose, in short, is to promote the teaching of phraseology in the Spanish as a foreign language classroom through literature.

Palabras

clave: phraseology; literature; Spanish as a foreign language; teaching/learning; Eduardo Mendoza. 


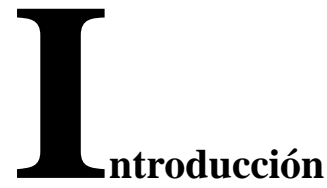

En el ámbito de la lingüística aplicada a la enseñanza del español como lengua extranjera, el debate sobre la importancia de incorporar las unidades fraseológicas en el aula se ha ido ampliando en los últimos años, abarcando no solo la conveniencia de incorporar estas unidades al proceso de enseñanza/aprendizaje, sino incluso otros aspectos específicos como qué tipología de unidades proponer, en qué nivel del proceso introducirlas, a través de qué tipo de actividades presentarlas, etc.

En la didáctica del español como lengua extranjera, el léxico se consideró durante décadas un elemento secundario comparado con la gramática. Sin embargo, si hasta la década de los años ochenta del siglo pasado el léxico se aprendía a través de listas de palabras que había que memorizar, a partir de la segunda mitad de los años ochenta se pasó a conceptualizar su aprendizaje como un conjunto de unidades relacionadas de manera diversa (Morante Vallejo, 2005).

A partir del Enfoque Léxico de Lewis (1993, 1997), la orientación ha ido cambiando todavía más, centrándose en el estudio del léxico y - en especial en los últimos años-, en el estudio del léxico 
desde una perspectiva combinatoria. A este cambio de conceptualización se ha asociado la explicitación de objetivos y estrategias que ayudaran a los aprendientes a ser conscientes tanto de la finalidad de lo que aprenden como de los medios empleados para conseguir los objetivos de aprendizaje. En este sentido, han desempeñado un papel importante los planteamientos de tipo constructivista (Coll, 1993; Gimeno y Pérez Gómez, 1992; Williams y Burden, 1997), que han desplazado el foco de atención desde la mera transmisión unidireccional de conocimientos hacia la co-construcción de estos por parte de los aprendientes. Tales enfoques han fomentado la autonomía del aprendiente en el proceso de aprendizaje y su participación activa. Hacia mediados de los años ochenta, además, la lingüística cognitiva (Langaker, 1987) recalcó la interrelación entre los niveles sintáctico, semántico y fonológico; se trata de una concepción totalmente innovadora de la estructura del lenguaje y, por lo tanto, de la investigación linguiística, ya que plantea que la gramática crea un continuum con el léxico y que puede ser descrita en términos de unidades simbólicas (formas-significados), sin desligarse de la semántica.

Tanto el Enfoque Comunicativo (Nunan, 1996) como el Enfoque Natural (Richards y Rodgers, 1986; Sánchez, 1997) contribuyeron a que se superasen las listas de palabras a favor de un aprendizaje significativo, a pesar de no proponer actividades explícitas de aprendizaje del léxico. El Enfoque Léxico, además de proponer la enseñanza explícita del léxico — sin por ello despreciar el aprendizaje implícito - fue importante porque sugirió que este nivel del sistema lingüístico -y no solo la gramática— supone un proceso de aprendizaje complejo, que requiere metodologías específicas, materiales adecuados y estrategias didácticas definidas. Lewis (1997, p. 7) señaló la importancia de aprender cómo se combinan las palabras entre ellas, apuntando a la fraseología en general y a las colocaciones y a las expresiones idiomáticas en particular, y recalcando que adquirir léxico significa organizar el lexicón mental, ya que, al cumplir este proceso, se integran nuevos conceptos y nuevas redes de significado y etiquetas para estos conceptos. 
El Diccionario de la lengua española de la Real Academia Española define la fraseología, en su quinta acepción, como 'Parte de la lingüística que estudia las frases, los refranes, los modismos, los proverbios y otras unidades de sintaxis total o parcialmente fija'. El español es una lengua muy rica en combinaciones de palabras, que suelen emplearse cotidianamente por parte de los nativos para comunicarse, y esta es la razón por la cual deberían ser presentadas en el aula de ELE. El objetivo de este trabajo es señalar la importancia de presentar las unidades fraseológicas en el aula de ELE mostrando una experiencia de aula. La pregunta a la que pretendemos responder es si la literatura puede representar un instrumento útil incluso para la enseñanza de las unidades fraseológicas en aula de ELE y, en caso afirmativo, a través de qué tipo de actividades; otro propósito es ampliar el acervo de actividades para trabajar estas unidades en el campo de la enseñanza/aprendizaje de la lengua extranjera. Una vez descritos brevemente desde un punto de vista teórico los ámbitos de la fraseología y de la literatura en la didáctica de ELE, se presenta la propuesta basada en la novela Sin noticias de Gurb, de Eduardo Mendoza, proporcionando unos ejemplos de actividades y técnicas concretas.

\section{Bases teóricas}

Antes de describir nuestra propuesta de actividades en el aula, consideramos necesario esbozar — aunque sea brevemente - las bases teóricas sobre las que se fundamentan las tendencias metodológicas adoptadas. Por este motivo, en los epígrafes siguientes la atención se dirigirá hacia la fraseología y el empleo de la literatura como instrumentos para el desarrollo de las competencias lingüística y comunicativa de los aprendientes en el aula de ELE.

\section{1. La fraseología en la didáctica de ELE}

Los motivos para enseñar las unidades fraseológicas son diversos: se trata de expresiones muy frecuentes, que aparecen a 
menudo en el lenguaje cotidiano, indistintamente del registro empleado; entre ellas, hay muchas opacas, es decir, cuyo significado no puede deducirse a partir de cada elemento que compone la expresión y, por lo tanto, no son fácilmente interpretables. Otro aspecto es el alto nivel expresivo que las caracteriza, que permite resumir en una única frase algo que posiblemente necesitaría explicaciones más extensas para ser expresado. El aspecto cultural tampoco es secundario, ya que a menudo se trata de unidades que conllevan un contenido cultural que ayuda a los aprendientes a comprender más a fondo el país cuya lengua están aprendiendo. Además, están relacionadas en muchas ocasiones con el lenguaje oral y coloquial. Que el estudiante sepa introducirlas en sus discursos hará que "se integre mejor en el medio nativo en el que se desenvuelva" (Saracho Arnáiz, 2016, p. 924). Otro motivo es que no se encuentran siempre en los diccionarios o en los manuales. Saracho Arnáiz (2016, p. 926) ha llevado a cabo una investigación en la cual analiza la presencia de las unidades fraseológicas en dieciséis manuales de ELE y ha llegado a la conclusión de que para los niveles iniciales (A1 y A2) suelen tratarse de forma copiosa tan solo las fórmulas rutinarias y que muy pocos presentan las colocaciones, mientras que las "unidades idiomáticas", es decir, las locuciones idiomáticas de distinto tipo (nominales, adjetivas, verbales, adverbiales...), aparecen solo en un manual, y además son escasas. La conclusión del análisis de Saracho es que el contenido fraseológico más completo y con presencia de actividades fraseodidácticas aparece en los manuales de nivel C. Esto supone que, para los niveles inferiores, el profesor de ELE tiene que tratar este tipo de contenido de forma casi autónoma y para ello necesita aportar materiales y actividades. También Ruiz Gurillo (2000) llega a la conclusión de que en los manuales de ELE la fraseología no se ha tratado con la profundidad adecuada.

A pesar de no ser una tarea exenta de dificultades, es oportuno que el profesor de ELE conozca las diferentes unidades fraseológicas de la lengua española para que pueda decidir qué tipo de unidades presentar en el aula y cuál es la metodología más adecuada para tratarlas. De hecho, como señala Corpas Pastor (1996, p. 32), "[s]i bien no parece haber acuerdo acerca de cuáles sean las unidades que comprende la fraseología y el término abarcador de todas ellas, tampoco 
se puede decir que exista mayor consenso a la hora de clasificar estos fenómenos léxicos".

En una primera aproximación, aquí consideraremos unidades fraseológicas las combinaciones de palabras con un nivel de fijación muy alto en su forma y en su significado, lo cual significa que, para que se dé una unidad fraseológica, la combinación de palabras tiene que ser estable o fija. Penadés Martínez señala que se trata de un concepto muy amplio (1999):

[...] dentro del concepto recubierto por el término unidad fraseológica o fraseologismo se incluye tanto lo que tradicionalmente se ha denominado dichos, expresiones fijas, expresiones idiomáticas, expresiones sin más, frases, modismos, giros, fórmulas, y también fórmulas proverbiales o fórmulas comunicativas, idiotismos, locuciones, modos de decir, frases hechas, refranes, adagios, proverbios o aforismos, como lo que más modernamente se ha llamado colocaciones, expresiones o unidades pluriverbales, lexicalizadas o habitualizadas y unidades léxicas pluriverbales (p. 13).

Diferentes taxonomías han propuesto autores como Zuluaga (1980), Higueras García (1997), Ruiz Gurillo (1997), que han contribuido a la clasificación de estas unidades. A pesar de las distintas clasificaciones que han llevado aparejadas distintas nomenclaturas, nos parece que la de Corpas Pastor es la más abarcadora. La estudiosa afirma que (1996):

las unidades fraseológicas (UFS) —objeto de estudio de la fraseología - son unidades léxicas formadas por más de dos palabras gráficas en su límite inferior, cuyo límite superior se sitúa en el nivel de la oración compuesta. Dichas unidades se caracterizan por su alta frecuencia de uso y de coaparición de sus elementos integrantes; por su institucionalización, entendida en términos de fijación y especialización semántica; por su idiomaticidad y variación potenciales; así como por el grado en el cual se dan todos estos aspectos en los distintos tipos (p. 20).

Entre los aspectos definitorios enumerados por Corpas Pastor, quizás el más complejo sea la idiomaticidad, es decir, "aquella propiedad semántica que presentan ciertas unidades fraseológicas, por 89 | Pá g i n a 
la cual el significado global de dicha unidad no es deducible del significado aislado de cada uno de sus elementos constitutivos" (Corpas Pastor, 1996, p. 26). Si tradicionalmente el aspecto idiomático se consideraba un aspecto esencial de una unidad fraseológica, en realidad, las unidades fraseológicas "pueden presentar dos tipos de significado denotativo: significado denotativo literal y significado denotativo figurativo o traslaticio, es decir, idiomático." (Corpas Pastor, 1996, p. 27). Así la denominación unidad fraseológica es amplia y engloba tanto expresiones transparentes como opacas. Por ejemplo, según Zuluaga (1980), las expresiones idiomáticas constan de un mínimo de dos palabras, cuya secuencia - gramatical o léxico-semántica- se mantiene estable en el tiempo, y presentan la idiomaticidad como característica, es decir, el "rasgo semántico propio de ciertas construcciones lingüísticas fijas, cuyo sentido no puede establecerse a partir de los significados de sus elementos componentes ni del de su combinación" (Zuluaga, 1980, pp. 121-134). En cambio, las colocaciones -que Higueras García (1997, p. 36) define como "combinaciones sintagmáticas" — suelen ser mucho más transparentes desde un punto de vista semántico y, a pesar de tener cierto nivel de estabilidad y mostrar cohesión interna, son menos fijas y admiten una mayor variación.

Basándose en la combinación de dos criterios, el de enunciado "- - y, por consiguiente, de acto de habla - con el de fijación (en la norma, en el sistema o en el habla)", Corpas Pastor (1996, p. 50) plantea un primer nivel de clasificación de las unidades fraseológicas en tres esferas. A este respecto, es importante aclarar que por enunciado se entiende "una unidad de comunicación mínima, producto de un acto de habla, que corresponde generalmente a una oración simple o compuesta, pero que también puede constar de un sintagma o una palabra" (Corpas Pastor, 1996, p. 51). Esto hace que se definan dos grupos distintos de unidades fraseológicas: las que no constituyen enunciados completos, y las que sí lo hacen. De este modo, en el primer grupo entran las unidades que no constituyen actos de habla ni enunciados y que, por lo tanto, requieren la combinación "con otros signos linguiísticos y que equivalen a sintagmas" (Corpas Pastor, 1996, p. 51). En este grupo, hay dos esferas: en la primera, entran diferentes unidades, como las 
colocaciones, es decir, unidades fijadas en la norma; y, en la segunda, las locuciones, que son unidades del sistema. En el segundo grupo, Corpas Pastor (1996, p. 51) introduce la esfera III y engloba en ella "aquellas UFS que pertenecen exclusivamente al acervo socio-cultural de la comunidad hablante (es decir, son unidades del habla)", que Corpas Pastor define como "enunciados fraseológicos". Se trata de unidades que están fijadas en el habla y que constituyen actos de habla "realizados por enunciados completos, dependientes o no de una situación específica" (Corpas Pastor, 1996, p. 51). Resumiendo, en la esfera I entrarían las colocaciones, en la esfera II las locuciones, y en la esfera III las paremias (citas, refranes) y las fórmulas rutinarias. En la actividad didáctica que se llevó a cabo y aquí presentamos, nos centramos en algunas de las unidades que entran en la esfera I y II. No obstante, nos interesamos especialmente por aquellas locuciones y expresiones idiomáticas cuyo significado, a diferencia de otras combinaciones de palabras, no es construido, sino que se compone en bloque a partir de diferentes procedimientos (Jezek, 2011, p. 198).

Así, al profesor de ELE se le requiere conocer diversos aspectos de la fraseología teórica y la tipología de estas unidades y que efectúe una reflexión profunda sobre cómo incorporarlas al proceso de enseñanza/aprendizaje. El Marco Común Europeo de Referencia (MCER) del Consejo de Europa (2002) define la competencia léxica como el conocimiento del vocabulario de una lengua, que se compone de elementos léxicos y elementos gramaticales, y la capacidad para utilizarlo. Los elementos léxicos o unidades léxicas comprenden: a) Unidades fraseológicas o unidades pluriverbales (expresiones hechas, modismos, estructuras fijas, etc.), que se componen de varias palabras que se emplean y se aprenden como un todo; b) Polisemia: una palabra puede tener varios sentidos distintos (MCER, 2002, p. 108). La discusión sobre el nivel en el que deberían introducirse en el diseño curricular estas expresiones es conocida. El MCER propone presentar algunas unidades (pensamos en las normas de cortesía y otras fórmulas rutinarias y fijas, por ejemplo) en niveles inferiores, en el A1, mientras que la mayoría de las expresiones (las expresiones idiomáticas, también denominadas modismos o frases hechas) aparecen casi exclusivamente en el nivel más alto, a partir del C1. Lo mismo ocurre para las 
expresiones de la sabiduría popular (refranes y otras unidades fraseológicas). Sin embargo, en los últimos años, han sido varias las propuestas para introducir estas unidades incluso en los niveles inferiores (Saracho Arnáiz, 2016), seleccionándolas según su dificultad, sobre todo, si el contexto de enseñanza es el de lenguas próximas (Minervini, 2019). En definitiva, concordamos con Muñoz-Basols (2015, p. 449) cuando afirma que, siguiendo unas pautas determinadas, el lenguaje idiomático puede integrarse en todos los niveles de aprendizaje.

\section{2. La literatura en la didáctica de ELE}

Una vez ilustrados los presupuestos teóricos en los que se basa nuestra idea de fraseología y tras haber expuesto los motivos por los cuales consideramos fundamental que a las unidades fraseológicas se les dé un tratamiento específico en la clase de ELE, a continuación se describen los motivos por los cuales pensamos que la literatura puede desarrollar una función destacada para conseguir el objetivo de enseñar las unidades fraseológicas.

Como es bien sabido, el empleo de la literatura en la enseñanza de las lenguas extranjeras ha sufrido variaciones a lo largo del tiempo. En el siglo XVIII, las lenguas modernas entraron en el currículo de las escuelas europeas, y el enfoque que se empleó para enseñarlas seguía fundamentalmente las pautas del estudio del latín. En este modelo, la gramática y su dominio adquirieron un papel fundamental, junto con la práctica de la traducción. El objetivo era memorizar las reglas de la lengua para manipular su morfología y su sintaxis, y el texto literario se convirtió en el elemento principal para este tipo de enseñanza, ya que representaba el modelo insigne y correcto de la lengua que se aprendía. Sin embargo, los textos literarios se estudiaban fuera de contexto, privándolos en muchas ocasiones de su sentido y empleándolos para enseñar reglas gramaticales. La influencia del denominado Método Tradicional (o de gramática-traducción) siguió presente durante décadas, hasta que, a finales del siglo XIX, el Movimiento de Reforma cambió las prioridades de la enseñanza. Con el Método Directo, se 
replantea el papel que se le otorga a la gramática dentro del proceso de enseñanza, al considerar que el aprendizaje de una lengua extranjera se logra como el de la lengua materna, es decir, de forma inductiva. Se hace hincapié en la lengua oral para llegar a comunicarse y se fija como uno de los objetivos principales aprender y usar la lengua como en situaciones de la vida cotidiana. Queda patente que, con este cambio de paradigma y con el empuje de los modelos estructurales a partir de los años sesenta y comunicativos en los años noventa del siglo pasado, no quedaba espacio para la literatura en los programas de enseñanza. La prioridad, de hecho, era comunicarse, procurando emplear la lengua usada en la vida cotidiana, y el texto literario se consideró muy alejado o, por lo menos, no adecuado para este propósito. Sin embargo, en las últimas décadas, el papel del texto literario en el aula de ELE se ha revalorizado: la literatura ya no representa —como se creía anteriormente - solo un modelo lingüístico de referencia, sino que ofrece múltiples elementos para ser explotados en el aula; de tipo lingüístico, pero también discursivo, pragmático y cultural' ${ }^{1}$ Se trata, pues, de un instrumento para aprender las claves culturales necesarias que facilitan las interacciones con hablantes nativos (Acquaroni, 2007, p. 15), y a esto hay que añadir que el texto literario no tiene por qué presentar un modelo de lengua alejado de los usos comunes:

Es evidente que el texto literario, necesariamente, se manifiesta siempre como resultado de un conjunto de usos de la lengua, pues su dimensión lingüística es obvia. Para el empleo de materiales literarios en el contexto curricular y de aula de LE, hay que tener muy presente que ni la literatura ni el discurso literario son necesariamente sinónimos de complejidad lingüística, ni de arbitrario desvío del uso de la lengua, porque lo cierto es que son

\footnotetext{
${ }^{1}$ Resulta interesante a este respecto el trabajo de Trovato $(2017$, p. 2$)$, en el que se presenta una panorámica sobre la didáctica de la lengua y la literatura en el contexto educativo italiano y en el que se describe el papel que puede desempeñar el texto literario dentro de un enfoque comunicativo y orientado a la acción. Trovato señala que, después de un periodo de 'abandono' de la literatura en la enseñanza secundaria en Italia, el texto literario ha vuelto a situarse en el primer plano en la enseñanza y el aprendizaje de los idiomas, representando "en un enfoque orientado a la acción, un soporte pedagógico irrenunciable a todos los niveles de aprendizaje de la lengua española, e incluso un punto de encuentro de las culturas y el espacio privilegiado de la interculturalidad".
} 
muchas las ocasiones que la producción literaria muestra la sencillez expositiva, la esencialidad gramatical y la claridad en su composición (Mendoza Fillola, 2008).

A esta revalorización de la literatura en el aula de ELE ha contribuido el dejar de considerar el texto literario como algo necesariamente alejado de la lengua 'real' para pasar a considerarlo un instrumento para desarrollar habilidades de comunicación, según Mendoza Fillola (2008):

(...) los textos literarios son una excelente cantera de materiales que aportan a través de la lectura un amplio, variado y enriquecedor input lingüístico y cultural, junto con otros conocimientos, datos y referencias válidos para la formación lingüístico-comunicativa del aprendizaje de LE. El texto literario es soporte y reflejo de los usos lingüísticos en sus funciones básicas de interrelación en la comunicación estética.

El empleo de la literatura para la formación lingüística y comunicativa en LE se basa en tres razones (Mendoza Fillola, 2008):

a. en primer lugar, la combinación y contraste del uso literario y del uso estándar de la lengua que se da en muchos textos, de modo que se unen los diferentes usos;

b. el continuum de usos, códigos y expresividad que funciona como input para la formación; y

c. la lectura como una actividad base para el aprendizaje; mediante la lectura se franquea el acceso al discurso literario y, especialmente, se favorece la observación de la continuidad de usos lingüísticos que en él aparecen.

En nuestra actividad, el empleo del texto literario no se ha concebido como estudio de la literatura en sí, que hubiera requerido otros procedimientos y el desarrollo de otras destrezas, sino que ha constituido un material dirigido a realizar actividades linguísticas y a desarrollar el conocimiento sociocultural del aprendiente. Sin embargo, esto no es óbice para que la lengua y la literatura se aprendan a la vez, ya que "lo deseable sería tratar de imbricar la lengua y la literatura, defender la conexión lengua-literatura como función formativa y aprendizaje lingüístico-comunicativo. En suma, verlo como algo complementario: aprender lengua y aprender literatura" (Sáez, 2011, p. 
59). De hecho, en el MCER (2002, p. 60) se afirma que "[1]os estudios literarios cumplen muchos más fines educativos, intelectuales, morales, emocionales, lingüísticos y culturales que los puramente estéticos".

Una vez que se ha asumido que el texto literario puede ayudar a desarrollar la competencia comunicativa del aprendiente y a acercarlo a la cultura de la lengua meta y a la lengua en contexto, en uso, otro interrogante al que debe responder el profesor de ELE es en qué nivel del proceso conviene introducir este tipo de material. Son varios los estudiosos que sugieren emplearlos ya desde los niveles iniciales, en concreto, a partir de un nivel A2. De hecho, como sugiere Sáez (2011, p. 61), estos textos tienen cabida en todos los niveles, si se aplican "criterios de lecturabilidad o grado de facilidad de lectura (léxico, longitud de frases, densidad semántica, etc.)".

Acquaroni (2007, p. 26) considera los géneros literarios desde un punto de vista discursivo "como conjuntos, relativamente estables y reconocibles, de técnicas expositivas vinculadas a determinadas leyes formales y de contenido, que se conforman históricamente y que poseen una intencionalidad comunicativa concreta". A pesar de que en este trabajo mostraremos algunas actividades para el desarrollo de la competencia fraseológica del aprendiente, decidimos emplear textos literarios en el aula de ELE porque pensamos que estos exhiben "diversas tipologías textuales, variedad de registros, contenidos socioculturales y lo implican [al alumno] emocionalmente al tratar temas y situaciones que puede extrapolar a su vida personal [...]" (Granero Navarro, 2017, p. 66). No obstante, nuestra intención ha sido que los aprendientes conceptualizaran la novela propuesta como un conjunto comunicativo más que como instrumento para el análisis lingüístico, es decir, como un mecanismo repleto de "formas y funciones del habla cotidiana" (Sáez, 2011, p. 60), muchas veces rebosante de expresividad y de variedad de los usos de la lengua. En este sentido, la novela Sin noticias de Gurb cumple perfectamente los requisitos.

Resumiendo, pensamos que el texto literario adecuadamente seleccionado representa un recurso valioso para la enseñanza de las 
unidades fraseológicas por los motivos siguientes: 1. las unidades se presentan en un contexto de uso real de la lengua y favorecen el desarrollo de la competencia pragmática; 2. aparecen expresiones pertenecientes a diferentes registros; en caso de aparecer un único registro, este permite igualmente una reflexión contrastiva sobre registros diferentes; 3. permiten ahondar en los elementos culturales; 4. los aprendientes se conciencian sobre la elevada densidad de estos elementos en la lengua meta (y en su lengua materna).

\section{Metodología}

No se pretende proponer aquí ninguna teoría metodológica sistematizada, sino proporcionar unos ejemplos de actividades y técnicas para trabajar las unidades fraseológicas en el aula de ELE a través de la literatura. Sin embargo, antes de presentar algunas estrategias concretas, nos parece necesario señalar que el texto se ha explotado a través de actividades didácticas variadas, cuyos objetivos eran diversos y más generales: el desarrollo de la competencia discursiva (a través, por ejemplo, de los mecanismos de cohesión y de organización retórica), la observación de otros contenidos lingüísticos (como el léxico coloquial y el uso de los verbos del pasado), la reflexión sobre los elementos culturales y sociolingüísticos (las comidas españolas, el ocio, la organización de la ciudad y sus problemas), etc. Se ha intentado seguir la recomendación de Acquaroni (2007, p. 46) y, a pesar de trabajar unos contenidos lingüísticos con fines didácticos, hemos procurado no depauperar el texto ni reducir "la emoción y el sentimiento a estructuras huecas".

\subsection{Elección de la novela}

Ventura Jorge (2015, p. 40) describe los tipos de textos que se emplean en la enseñanza de ELE (narrativos, dramáticos y poéticos) y señala que "entre ellos, la novela, el cuento, el microrrelato, el teatro y la poesía son los más utilizados". Todos ellos, a excepción de la novela, pueden ser introducidos en el aula fácilmente gracias a su corta 
extensión, que permite trabajar con ellos en una sesión, y se prestan "a una lectura intensiva cuya finalidad principal es que los alumnos amplíen su vocabulario a través de una lectura atenta y la realización en el aula de distintos tipos de actividades" mientras que la novela y el teatro "se prestan más a una lectura extensiva, con la que se pretende que los estudiantes comprendan el sentido general de la obra que están leyendo". En este trabajo, Ventura Jorge (2015: 43-46) también aborda el debate sobre si utilizar textos clásicos o actuales, así como sobre si es mejor usar textos originales, graduados o adaptados. En nuestro caso, hemos abogado por una novela, que permitía una lectura más extensiva, $\mathrm{y}$ un texto original y actual.

Los motivos que justifican la lectura de la novela de Eduardo Mendoza son múltiples: se trata de un texto muy expresivo, escrito en un estilo sencillo y en el que los coloquialismos ocupan un lugar destacado. Además, el uso de la ironía hace que la lectura sea amena y que los estudiantes puedan reírse. Sin embargo, se puede elegir cualquier tipo de texto literario, en especial de narrativa contemporánea, ya que, como sugiere Mendoza Fillola (2008), esta "posibilita amplias, diversas e interesantes actividades para desarrollar tanto aspectos genéricos del aprendizaje lingüístico y cultural como facetas específicas para el aprendizaje comunicativo". Además, como señala el mismo autor, se trata de textos que no suelen resultar más complicados de leer que un artículo periodístico o un folleto publicitario.

Sin noticias de Gurb apareció por entregas en el periódico y se publicó luego como novela en 1990; como escribe Mendoza en la nota al texto de la edición de 2014 (al que aquí hacemos referencia, p. 7), "no es en rigor un libro, o no nació con voluntad de serlo" y es una historia que nació "fraccionada" en entregas para el periódico El País. Su estructura, que prevé "la división del tiempo narrativo en fracciones mínimas" (p. 9), es muy útil porque hace que los aprendientes perciban el texto como "fácil de leer" (p. 10) y ágil. Las frases son breves y su estructura es simple. A esto contribuye también "su contenido ligero" (p. 10), a pesar de que en él aparezca una crítica, a veces liviana y otras despiadada, hacia la sociedad española, y de tratarse de - en palabras de su autor (p. 10) _ "un libro alegre" y lleno de humor. Es una novela 
de viajes en la que el protagonista llega a un nuevo planeta y a una nueva ciudad, la Barcelona preolímpica, y entra en contacto con sus habitantes y sus costumbres. Se trata de una situación que experimentamos un poco todos cuando viajamos, incluso nuestros estudiantes, y con la que se sentirán identificados y, a la vez, les despertará la curiosidad. Además, su lectura permite conocer varios ámbitos de la sociedad y de la cultura españolas a través de la ironía. Por lo que a las unidades fraseológicas se refiere, aparecen numerosas $\mathrm{y}$, entre ellas, varias pertenecen al registro coloquial y, a veces, incluso vulgar, característica que divertirá a los aprendientes.

\section{2. Contexto y propuestas prácticas}

En este epígrafe describimos el contexto de aula y algunas de las actividades desarrolladas. Los aprendientes que han participado en las actividades propuestas son estudiantes universitarios del curso de grado en Ciencias Políticas de la Universidad de Salerno (Italia) cuya lengua materna es el italiano y que estaban cursando su segundo año de español, es decir, desde un nivel B1 debían alcanzar un nivel B2. Además, estaban estudiando una segunda lengua extrajera en el ámbito universitario (inglés o francés). Estas actividades se han propuesto a dos grupos de estudiantes con un nivel parecido: un primer grupo estaba formado por 10 estudiantes; el segundo, por 11.

Al tratarse de aprendientes universitarios, nuestra metodología contemplaba una breve explicación teórica sobre las unidades fraseológicas: qué son, qué función desarrollan en la comunicación, cómo se emplean, etc. Este tipo de explicitación suele favorecer la reflexión sobre la importancia de estas unidades, pero también sobre la lengua materna de los aprendientes, ayudándoles a usarlas de forma más consciente. También consideramos conveniente explicar las razones por las que se presentan en el aula (el español es una lengua muy rica en unidades fraseológicas, los nativos suelen emplearlas mucho en la conversación cotidiana, ya que con ellas es posible expresar un concepto amplio empleando una menor cantidad de palabras, etc.), porque suelen motivar mucho a los aprendientes. 
Los primeros ejercicios propuestos iban dirigidos hacia la diferenciación de las distintas unidades fraseológicas, con el fin de que ya desde la primera lectura de la obra pudieran identificar una colocación (cometer un error/*hacer un error), una expresión idiomática (tener mala leche) y un refrán (En casa del herrero, cuchara de palo). Para fomentar su participación activa, se les pidió que, a medida que fueran leyendo el libro, apuntaran las expresiones que consideraban colocaciones, locuciones o expresiones idiomáticas para posteriormente debatir sobre ellas en cada clase y decidir en qué tipología incluirlas. La novela se leyó a lo largo de todo el curso, un semestre universitario, y se les pidió a los estudiantes que leyeran en casa la parte que se iba a debatir en la clase siguiente. También debían contestar a una serie de preguntas que servían para desarrollar su comprensión del texto escrito y ampliar su conocimiento sociocultural de España.

Entre las unidades propuestas por los estudiantes, encontramos colocaciones (alta densidad, establecer contacto, fruncir el ceño, armario empotrado, blanquear dinero negro, gastar un dineral, análisis somero), locuciones sustantivas (quebradero de cabeza), locuciones verbales (matar el tiempo), locuciones adverbiales (en un santiamén, como un tiro, de sol a sol, a cal y canto), adjetivas (ciudadano de a pie) e idiomáticas (tener monos en la cara). Si algunas unidades son fáciles de catalogar (pensamos en alta densidad o establecer contacto, que son claramente colocaciones), otras, en cambio, suponen un mayor desafío. De hecho, dinero negro es una colocación para la mayoría de los estudiosos (Corpas Pastor, 1996, p. 73), mientras que expresiones como fruncir el ceño o matar el tiempo son catalogadas como colocaciones por algunos autores y locuciones por otros. Consideramos importante, en todo caso, que los estudiantes entiendan el valor 'unitario' de la expresión, aunque no siempre sepan catalogarla del todo correctamente. Tras clasificar por tipologías las unidades que habían encontrado durante las clases, el segundo ejercicio que se propuso consistió en que las parafrasearan con el objetivo de averiguar si habían entendido su significado explicándolo con otras 
palabras. En el ejemplo 1, se ilustran algunas paráfrasis propuestas por lo estudiantes:

\section{Ejemplo 1}

Tener monos en la cara: cuando alguien nos mira raro y no sabemos el motivo.

Dormir la mona: tener resaca; sensaciones relacionadas con la borrachera.

Entrarle por los ojos: gustar a primera vista.

Caer en saco roto: olvidar o no tener algo en cuenta.

Dar la lata: fastidiar o molestar.

Para aclarar el significado, también se les planteó y resultó útil una actividad en la que tenían que relacionar cada expresión con la definición correspondiente, como en el ejemplo 2:

\section{Ejemplo 2}

1. Sumir(se) en un mar de confusiones.

2. Dormir a pierna suelta.

3. Pasar la noche del loro.

4. Poner a alguien de vuelta y media.

5. Ver cómo está el patio.

6. Ser un piernas. a. Averiguar la situación.

b. No conseguir dormir.

c. Dormir, descansar de un tirón.

d. Hacer comentarios ofensivos sobre alguien.

e. No tener las cosas claras.

f. No tener habilidades.

El paso siguiente consistió en buscar un equivalente en su lengua materna y señalar el registro de uso $^{2}$. De las tres técnicas

\footnotetext{
${ }^{2}$ Como se ha mencionado, a la hora de presentar las unidades fraseológicas, también es importante que se aclare a qué variante pertenecen. En nuestro caso, se ha obviado este paso, puesto que las expresiones no habían sido elegidas por el profesor, sino que se encontraban ya en el texto. De todos modos, consideramos que la elección debería estar guiada por los intereses de los aprendientes, considerando aspectos como su cercanía a un determinado país, así como la familiaridad del profesor con un determinado tipo de español. En el caso de estudiantes italianos de español, la elección 100 | Página 
(visuales, verbales y traducción) que Peña Portero (2005) sugiere para trabajar las expresiones idiomáticas, se emplearon la verbal y la traducción. Una vez comprendido el significado de la unidad se les explicó a los aprendientes que es conveniente efectuar su traducción en bloque y no palabra por palabra, debido a su frecuente carácter opaco. La traducción favorece una reflexión de tipo contrastivo con la lengua materna, que se ampliará posteriormente con una actividad dirigida a encontrar una expresión equivalente en italiano.

Para este objetivo, nos sirvió de guía Corpas Pastor (2000), que habla de diversos grados de equivalencia en el plano léxico por lo que a los binomios fraseológicos se refiere: total, parcial y nula. Entre las lenguas próximas son frecuentes las equivalencias totales, es decir, unidades fraseológicas que la estudiosa denomina europeísmos o calcos (Corpas Pastor 2003, p. 217). Son unidades que coinciden, ya que expresan el mismo significado denotativo y connotativo, y presentan una misma base metafórica, la misma frecuencia de uso, el mismo valor pragmático, etc. Luego existe la equivalencia aparente o falsos amigos (Corpas Pastor, 2003, p. 218), que se da cuando las dos estructuras son aparentemente equivalentes porque presentan semejanzas formales, pero contenidos semánticos distintos. Y, por último, están las que no tienen equivalencia idiomática y para las que es necesario recurrir a una explicación. Por lo que a las lenguas próximas se refiere, como las que aquí nos ocupan, Trovato (2018, p. 423) plantea para las expresiones idiomáticas un enfoque traductológico contrastivo con el que clasifica las expresiones que permite averiguar "cómo las dos lenguas expresan determinados conceptos, desde una perspectiva pragmática y cultural" y que se basa en la división de estas en tres secciones: de fácil inteligibilidad entre español e italiano, con un grado de dificultad medio y de difícil inteligibilidad.

En este momento del proceso, se les explicó a los aprendientes que entre italiano y español ${ }^{3}$ hay expresiones de equivalencia plena (cerrar el pico / chiudere il becco); de equivalencia parcial (estar hasta

sería la variante de España, tanto por la cercanía geográfica entre los dos países como por el tipo de formación.

${ }^{3}$ Sobre fraseología contrastiva, véase el trabajo de Navarro (2008). 
las narices / averne le scatole piene), es decir, con una base estructural distinta, pero con el mismo o parecido valor semántico-pragmático; y de equivalencia nula (en el quinto pino). Esta explicación les ayudó a buscar la traducción en su lengua materna y a fijar las expresiones. Algunas de las traducciones que propusieron se ilustran en el ejemplo 3:

\section{Ejemplo 3}

1. Llover a cántaros / piovere a catinelle (correspondencia total).

2. Dormir a pierna suelta / dormire della grossa / dormire il sonno dei giusti / dormire a quattro di bastoni (correspondencia parcial).

3. Poner a alguien de vuelta y media / dirne di tutti i colori di qualcuno (correspondencia parcial).

4. Poner los pelos de punta / far venire la pelle d'oca (correspondencia parcial).

5. Dar la lata / rompere le scatole (correspondencia parcial).

6. Acabar como el rosario de la aurora / finire a pesci in faccia / finire in baraonda (correspondencia parcial).

7. Dormir la mona / smaltire la sbornia (correspondencia parcial).

8. Tener monos en la cara / che c'è da guardare? (correspondencia nula).

9. Pasar la noche del loro / passare la notte in bianco (correspondencia parcial).

10. Dormir como un ceporro / dormire come un ghiro (correspondencia parcial).

Para practicar el tema del registro, se les pidió que parafrasearan las unidades que permitían un cambio de registro; en nuestro caso, se trataba de pasar del registro coloquial al formal, como se observa en el ejemplo 4:

\section{Ejemplo 4}

Estar más buena que el pan = Ser muy guapa, tener un cuerpo muy bonito.

Salir pitando = Salir con mucha prisa .

A mí, plin = No me interesa . 
Dar la lata = Importunar, molestar .

A medida que iban leyendo el libro y extrapolando las unidades, se les fue proponiendo otra actividad que consistía en emplear las que encontraban para formular frases nuevas con contextos adecuados. Se trata de una fase de producción libre, no controlada, útil, sobre todo, para averiguar si se han entendido el registro correcto de cada expresión y las situaciones comunicativas en las que puede emplearse. Se les pidió que escribiesen la misma frase, pero adoptando un registro más formal. También en este caso proporcionamos algunos ejemplos de las propuestas de los aprendientes:

\section{Ejemplo 5}

Llover a cántaros:

Lo siento, no puedo pasar por tu casa porqué [sic] está lloviendo a cántaros.

Lo siento, no puedo pasar por tu casa porqué [sic] está lloviendo mucho.

Se suponía que íbamos a hacer una excursión de montaña, pero estuvo lloviendo a cántaros todo el fin de semana.

Se suponía que íbamos a hacer una excursión de montaña, pero estuvo lloviendo muchísimo todo el fin de semana.

Tener monos en la cara:

Odio cuando alguien me mira como si tuviera monos en la cara. Odio cuando alguien me mira directamente a la cara sin ninguna razón.

¿Por qué me miras? ¿Tengo monos en la cara?

¿Por qué me miras? Deja de mirarme tan fijamente.

Poner a alguien de vuelta y media:

Cuando no estoy, Alonso siempre me pone de vuelta y media con las demás personas.

Cuando no estoy, Alonso siempre me critica con las demás personas. 
Ponerse los pelos de punta:

Ayer fui al cine con Miguel para ver una película de terror, pero no la vimos toda porque teníamos miedo de volver a casa después. Las películas de terror me ponen los pelos de punta.

Ayer fui al cine con Miguel para ver una película de terror, pero no la vimos toda porque teníamos miedo de volver a casa después. Las películas de terror me dan miedo.

Cuando el león en el corral me miró, se me pusieron los pelos de punta. Cuando el león en el corral me miró, me asusté mucho.

Dar la lata:

Desde que Luis compró su nueva guitarra, no deja de darnos la lata con la musiquilla a todas las horas del día.

Desde que Luis compró su nueva guitarra, no deja de molestarnos con la musiquilla a todas las horas del día.

Incluso hubo casos en los que emplearon de forma autónoma otra expresión coloquial, como en los ejemplos en los que aparecen subir las escaleras a cuatro patas o no pegar ojo:

Dormir la mona:

Manuel bebió toda la noche en el bar de su amigo porque su novia lo dejó. Apenas llegó a casa, subió las escaleras a cuatro patas, se puso [sic] en nuestro sofá recién tapizado y durmió la mona. La mañana después no recordaba nada.

Manuel bebió toda la noche en el bar de su amigo porque su novia lo dejó. Apenas llegó a casa, subió las escaleras a cuatro patas, se puso [sic] en nuestro sofá recién tapizado y durmió un sueño profundo. La mañana después no recordaba nada.

Creo que deberías venir a dormir la mona a mi casa.

Creo que deberías venir a ponerte sobrio a mi casa. 
Pasar la noche del loro:

Los padres en paro, que están afectados por la durísima crisis que atravesamos, pasan la noche del loro porque no tienen nada para dar de comer a sus hijos.

Los padres en paro, que están afectados por la durísima crisis que atravesamos, no pegan ojo por la noche porque no tienen nada para dar de comer a sus hijos.

Los padres en paro, que están afectados por la durísima crisis que atravesamos, pasan la noche sin dormir porque no tienen nada para dar de comer a sus hijos.

Ayer Juan pasó una noche del loro porqué [sic] tenía demasiados malos pensamientos.

Ayer Juan no durmió porqué [sic] tenía demasiados malos pensamientos.

Ana ha tenido [sic] dolor de cabeza y ha pasado una noche del loro. Ana ha tenido [sic] dolor de cabeza y ha pasado toda la noche sin pegar ojo.

Después de la operación pasé una noche del loro.

Después de la operación pasé toda la noche sin pegar ojo.

Estos son solo algunos ejemplos de las posibilidades de las que dispone el profesor de ELE para trabajar las unidades fraseológicas en el aula. También pueden emplearse otras técnicas, como los ejercicios de respuesta múltiple en los que se presentan las unidades contextualizadas, las técnicas visuales, los ejercicios de rellenar huecos y de sustitución, así como el uso de diccionarios combinatorios como el Diccionario de locuciones verbales para la enseñanza del español (Penadés Martínez, 2002) o el Diccionario de locuciones nominales, adjetivas y pronominales para la enseñanza del español (Penadés Martínez, 2008), ambos organizados por nivel de enseñanza/aprendizaje, o los diccionarios Redes y el Diccionario combinatorio práctico del español contemporáneo: las palabras en su contexto (Bosque, 2004 y 2006), que representan instrumentos de trabajo esenciales para profundizar en los mecanismos combinatorios de las palabras del español. 


\section{Conclusiones}

En los últimos años, el interés hacia la enseñanza de las unidades fraseológicas en la clase de ELE se ha incrementado y, con ello, se ha reavivado el debate sobre qué tipo de expresiones presentar, en qué fase del proceso de enseñanza/aprendizaje, mediante qué actividades, etc. Somos conscientes de que la presentación de las unidades fraseológicas en el aula de ELE es una tarea a veces compleja, que requiere superar algunos obstáculos que van desde la formación del profesor hasta la comprensión del contexto de uso y del registro, y la memorización por parte de los aprendientes.

Sin embargo, pensamos que el trabajo con esta novela, además de los aspectos culturales, les ha permitido aprender vocabulario $\mathrm{y}$ expresiones fraseológicas en contexto $\mathrm{y}$, por lo tanto, su uso pragmático. Los estudiantes suelen mostrar curiosidad hacia estas expresiones y esto les motiva. Se debe posiblemente a que entender su significado en los casos de mayor opacidad es un juego, como resolver una adivinanza, y también porque perciben que aprendiéndolas entran en contacto con los usos reales de la lengua. Además, presentarlas a través de un texto literario como el de Mendoza facilita la comprensión del contexto de uso y de la identidad cultural del país cuya lengua se está aprendiendo, así como de otros elementos como el registro.

Finalmente, el empleo de estas unidades propicia que se desarrolle una reflexión sobre la lengua materna. Los ejemplos de actividades propuestos estaban orientados a estudiantes universitarios italianos de nivel B1 que ya habían trabajado con las unidades fraseológicas. Sin embargo, creemos que pueden adaptarse a otros contextos parecidos. Pensamos que el contexto es fundamental a la hora de aprender estas unidades y que la literatura desempeña un papel fundamental en su contextualización. La competencia fraseológica no consiste solo en usar segmentos fijos de la lengua; requiere que el estudiante conozca "muy bien la estructura externa de la unidad y su significado, y al mismo tiempo, debe saber adecuar la unidad perfectamente al texto y al contexto" (Saracho Arnáiz 2016, pp. 923- 
924). En este sentido, podemos afirmar que las actividades han dado buenos resultados, como demuestran incluso los obtenidos en los exámenes, y que el texto literario representa un instrumento utilísimo para ayudar al profesor de ELE en esta tarea compleja que es enseñar las unidades fraseológicas. Considerando la activa participación de los estudiantes y los resultados obtenidos, una futura línea de investigación podría ser la creación de otras actividades que puedan emplearse en la clase de ELE utilizando otras obras literarias de acuerdo con el nivel, los intereses y las necesidades de los estudiantes y del curso, incluso agrupándolas según criterios determinados, como pueden ser la frecuencia de uso, el registro, la variación diatópica y/o el significado.

\section{Referencias bibliográficas}

Acquaroni, R. (2007). Las palabras que no se lleva el viento: literatura y enseñanza del español como LE/L2. Madrid: SantillanaUniversidad de Salamanca.

Bosque, I. (dir.) (2004). Redes. Diccionario combinatorio del español contemporáneo. Madrid: SM.

Bosque, I. (dir.) (2006). Diccionario combinatorio práctico del español contemporáneo. Madrid: SM.

Coll, C. (1993). El constructivismo en el aula. Barcelona: Graó.

Consejo de Europa (2002). Marco común europeo de referencia para las lenguas: aprendizaje, enseñanza, evaluación. Madrid: Ministerio de Educación, Cultura y Deporte. Recuperado de https://cvc.cervantes.es/ensenanza/biblioteca_ele/marco/cvc_mer.pdf. Versión original: Common European Framework of Reference for Languages: Learning, Teaching, Assessment. Strasbourg: Council of Europe, 2001.

Corpas Pastor, G. (1996). Manual de fraseología española. Madrid: Gredos.

Corpas Pastor, G. (2000). Acerca de la (in)traducibilidad de la fraseología. En G. Corpas Pastor, (ed.), Las lenguas de Europa, estudios de fraseología, fraseografía y traducción (pp. 483-522). Granada: Comares. 
Corpas Pastor, G. (2003). Diez años de investigación en fraseología: análisis sintáctico-semánticos, contrastivos y traductológicos. Madrid: Iberoamericana.

Gimeno, J., y Pérez Gómez, A. (1992). Comprender y transformar la enseñanza. Madrid: Morata.

Granero Navarro, A. (2017). La literatura en clase de ELE. Actas del II Encuentro de Profesores de Español en Sofía, Bulgaria. Sofia: Instituto Cervantes, 64-71. Recuperado de https://cvc.cervantes.es/ensenanza/biblioteca_ele/publicaciones_centros /PDF/sofia_2017/07_granero.pdf.

Higueras García, M. (1997). Las unidades léxicas y la enseñanza del léxico a extranjeros. REALE, 8, 35-49. Recuperado de https://ebuah.uah.es/dspace/bitstream/handle/10017/7411/unidades_hig ueras_REALE_1997.pdf?sequence $=1$ \&isAllowed $=\mathrm{y}$.

Jezek, E. (2011). Lessico. Classi di parole, strutture, combinazioni. Bologna: Il Mulino.

Langaker, R. W. (1987). Foundations of Cognitive Grammar. Vol. I: Theoretical Prerequisites. London: Longman.

Lewis, M. (1993). The Lexical Approach. London: Language Teaching Publications.

Lewis, M. (1997). Implementing the Lexical Approach. London: Language Teaching Publications.

Mendoza, E. (1990, 2014). Sin noticias de Gurb. Barcelona: Seix Barral.

Mendoza Fillola, A. (2008). La utilización de materiales literarios en la enseñanza de lenguas extranjeras. Alicante: Biblioteca Virtual Miguel de Cervantes. Recuperado de http://www.cervantesvirtual.com/obra-visor/la-utilizacion-demateriales-literarios-en-la-ensenanza-de-lenguas-extranjeras--0/html/.

Minervini, R. (2019). Las expresiones idiomáticas en la enseñanza/aprendizaje de ELE: un estudio sobre el nivel intermedio (B1/B2), Rhesis, 10(1), 19-33. Recuperado de https://www.rhesis.it/wpcontent/uploads/Rhesis-LP-10.1-02-Rosaria-Minervini.pdf\#page=5.

Morante Vallejo, R. (2005). El desarrollo del conocimiento léxico en segundas lenguas, Madrid: Arco/Libros. 
Muñoz-Basols, J. (2015), Enseñanza del lenguaje idiomático. En Gutiérrez Rexach, J. (coord.), Enciclopedia de Lingüística Hispánica, 2, 442-453.

Navarro, C. (2008). Aspectos de fraseología contrastiva. Verona: Fiorini.

Nunan, D. (1996). El diseño de tareas para la clase comunicativa. Madrid: Cambridge University Press.

Penadés Martínez, I. (1999). La enseñanza de las unidades fraseológicas. Madrid: Arco/Libros.

Penadés Martínez, I. (2002). Diccionario de locuciones verbales para la enseñanza del español. Madrid: Arco/Libros.

Penadés Martínez, I. (2008). Diccionario de locuciones nominales, adjetivas y pronominales para la enseñanza del español. Madrid: Arco/Libros.

Peña Portero, A. (2005). Propuesta metodológica para enseñar expresiones idiomáticas. Redele 3. Recuperado de http://www.educacionyfp.gob.es/mc/redele/bibliotecavirtual/numerosanteriores/2005/memoriamaster/1-semestre/de-lapena.html.

Real Academia Española. Diccionario de la lengua española. 23. a ed., [versión 23.4 en línea]. Recuperado de https://dle.rae.es.

Richards, J., y Rodgers, T. (1986). Enfoques y métodos en la enseñanza de idiomas. Madrid: Cambridge University Press, 1998.

Ruiz Gurillo, L. (1997). Aspectos de fraseología teórica española. Valencia: Universitat de València.

Ruiz Gurillo, L. (2000). Un enfoque didáctico de la fraseología española para extranjeros (1), Espéculo. Recuperado de http://www.ucm.es/info/especulo/ele/fraseolo.html.

Sáez Martínez, B. (2011). Texto y literatura en la enseñanza de ELE. En J. de Santiago-Guervós, H. Bongaerts, J. J. Sánchez Iglesias y M. Seseña Gómez (coords.), Del texto a la lengua: La aplicación de los textos a la enseñanza-aprendizaje del español L2. Vol. 1, (pp. 57-68). Recuperado de https://dialnet.unirioja.es/servlet/articulo?codigo=5419236.

Sánchez, A. (1997). Los métodos en la enseñanza de idiomas. Evolución histórica y análisis didáctico. Madrid: SGEL. 
Saracho Arnáiz, M. (2016). Cómo desarrollar la competencia fraseológica en la clase de ELE. La formación y competencias del profesorado de ELE: XXVI Congreso Internacional ASELE, 921-931, Asociación para la Enseñanza del Español como Lengua Extranjera. Recuperado de https://cvc.cervantes.es/ensenanza/biblioteca_ele/asele/pdf/26/26_0921. pdf.

Trovato, G. (2017). DLL en el contexto educativo italiano: evolución histórico-metodológica, nuevas tecnologías y atención a la diversidad. Lenguaje $y$ textos, 45, 1-16. doi: http://doi.org/10.4995/lyt.2017.7412. Recuperado de https://polipapers.upv.es/index.php/lyt/article/view/7412/7820.

Trovato, G. (2018). Un estudio contrastivo y pedagógico español-italiano acerca del tratamiento lingüístico-traductológico de unidades léxicas dotadas de carácter figurado e idiomático. Orillas, 7, 417-428.

Recuperado de http://orillas.cab.unipd.it/orillas/articoli/numero_7/09Trovato_Astilleros .pdf.

Ventura Jorge, M. S. (2015). La literatura como recurso didáctico en la enseñanza del español como lengua extranjera. Tejuelo, 21, 30-53. Recuperado de http://dehesa.unex.es/handle/10662/4529.

Williams, M., y Burden, R. L. (1999). Psicología para profesores de idiomas. Enfoque del constructivismo social. Madrid: Cambridge University Press.

Zuluaga, A. (1980). Introducción al estudio de las expresiones fijas. Frankfurt: Peter Lang. 